\title{
Adaptive Gearshift Strategy Based on Generalized Load Recognition for Automatic Transmission Vehicles
}

\author{
Yulong Lei, ${ }^{1}$ Ke Liu, ${ }^{1}$ Yuanxia Zhang, ${ }^{1}$ Yao Fu, ${ }^{1}$ Hongbo Liu, ${ }^{2}$ Ge Lin, ${ }^{3}$ and Hui Tang \\ ${ }^{1}$ State Key Laboratory of Automotive Simulation and Control, Jilin University, Changchun 130025, China \\ ${ }^{2}$ Geely Group R\&D Center, Hangzhou 311200, China \\ ${ }^{3}$ China FAW Group Corporation R\&D Center, Changchun 130013, China
}

Correspondence should be addressed to Ke Liu; liuke12@mails.jlu.edu.cn

Received 28 January 2015; Revised 17 May 2015; Accepted 31 May 2015

Academic Editor: Dan Simon

Copyright (C) 2015 Yulong Lei et al. This is an open access article distributed under the Creative Commons Attribution License, which permits unrestricted use, distribution, and reproduction in any medium, provided the original work is properly cited.

\begin{abstract}
Recognizing various driving conditions in real time and adjusting control strategy accordingly in automatic transmission vehicles are important to improve their adaptability to the external environment. This study defines a generalized load concept which can comprehensively reflect driving condition information. The principle of a gearshift strategy based on generalized load is deduced theoretically, adopting linear interpolation between the shift lines on flat and on the largest gradient road based on recognition results. For the convenience of application, normalization processing is used to transform generalized load results into a normalized form. Compared with the dynamic three-parameter shift schedule, the complex tridimensional curved surface is not needed any more, so it would reduce demands of memory space. And it has a more concise expression and better real-time performance. For the target vehicle, when driving uphill with gradient $11 \%$, the vehicle load is about $280 \sim 320 \mathrm{Nm}$; when driving downhill, the value is around $-340 \sim-320 \mathrm{Nm}$. Road tests show that generalized vehicle load keeps near 0 in zero-load condition after calibration, and an $11 \%$ grade can be estimated with less than $1.8 \%$ error. This method is convenient and easy to implement in control software and can identify the driving condition information effectively.
\end{abstract}

\section{Introduction}

The effects of various driving conditions, such as grade, vehicle loading, and road resistance, on the powertrain control strategy should be considered carefully. For instance, the grade resistance increases during uphill driving, so a large transmission ratio should be selected to avoid frequent shifting. A large ratio should also be used during downhill driving to take full advantage of the engine braking effect and avoid gear shift-up. Similarly, aerodynamic resistance and rolling resistance increase during vehicle loading, so a large ratio should still be used to improve vehicle dynamic performance. Therefore, automatic transmission vehicles should recognize the aforementioned driving conditions in real time and adjust the corresponding shift algorithm to improve the vehicle's dynamic, passing, and comfort performance. Driving environment recognition is a prerequisite in achieving intelligent control.
Many driving environment recognition algorithms are available. For instance, Yuhai et al. [1] and Jin et al. [2, 3] developed certain methods to calculate the grade using an equation deduced by the principle of vehicle system dynamics. Ohnishi et al. [4] utilized an additional sensor, and Jo et al. [5] used GPS to identify the ramp and load, which will increase costs in practical application. Parameter identification is widely used to identify the driving environment [6-8], which not only depends on some vehicle parameters but also requires additional vehicle sensors. In addition, the process of real-time parameter estimation requires the electronic control unit (ECU) to have a higher computing speed. Another commonly used method is based on the fuzzy logic inference model [9-13], where the fuzzy rule can be flexibly adjusted according to the actual application situation. However, recognition results are generally the judgment and classification of the current vehicle condition instead of the precise slope or vehicle load. 
Notably, Hebbale et al. [14] and Bai et al. [15] introduced a method using the difference between the vehicle actual acceleration and nominal vehicle model (i.e., when driving on a flat and suitable asphalt concrete road with no load) acceleration to reflect the current vehicle load. According to the classic longitudinal vehicle dynamics equation [16], this study defines a generalized vehicle load concept based on the torque difference, which can comprehensively reflect driving condition information, such as the grade, loading mass, aerodynamic resistance, and rolling resistance. A corresponding generalized load recognition method is introduced, and its basic principle and factors that affect recognition result in different driving conditions are described and analyzed. The principle of a gearshift strategy based on generalized load is deduced theoretically. And linear interpolation is adopted to get corresponding shift lines under different driving condition. This method is adaptive to the general driving environment. Compared with the dynamic three-parameter shift schedule, the complex three-dimensional surface is not needed any more, so it would reduce demands of memory space. And it has a more concise expression and better realtime performance. Real vehicle tests showed this method is convenient and easy to implement in control software, the busy-shift phenomenon on the slope road can be eliminated, and drivers' dynamic requirement can be satisfied.

\section{Definition of Generalized Vehicle Load}

2.1. Definition. The general driving environment is a combination of different environmental factors in the vehicle driving-resistance balance equation [16], including ramp, load, weather, and road conditions. Therefore, the essence of general driving environment recognition is recognizing automobile driving resistance. Vehicle load generally refers to the mass of cargos or passengers, and this study extends this concept on the basis of the vehicle dynamics equation. We define a special zero-load driving condition and generalized vehicle load.

Zero-load driving condition refers to driving of a no-load vehicle on a flat, straight, and suitable asphalt concrete road in normal weather without braking.

Generalized vehicle load (or vehicle load) is defined as the force difference between the vehicle driving force in current driving conditions and resistances in zero-load condition when driving with the same speed and acceleration. This factor can be expressed in the following equation on the basis of the classic longitudinal vehicle dynamics equation:

$$
L_{N}=F_{t}-\left(F_{f z}+F_{w z}+F_{j z}\right)
$$

where $L_{N}$ is the vehicle load in unit $\mathrm{Nm}, F_{t}$ is the current vehicle driving force, $F_{f z}$ is the rolling resistance in zeroload condition, $F_{w z}$ is the aerodynamic resistance in zeroload condition, and $F_{j z}$ is the accelerating resistance in zeroload condition.

Given the flat road, the grade resistance $F_{i}$ does not appear in (1). Generalized vehicle load reflects the sum of the outside driving resistance. The larger the generalized vehicle load, the more the vehicle power demand. Therefore, the generalized vehicle load also reflects the external environment demand of vehicle power.

2.2. Conversion of Vehicle Load Formula. Equation (1) is obtained by the direct transposition of the vehicle dynamics equation, which is in an easily understood form. However, the equation is further transformed to facilitate the following driving condition recognition operation.

Taking automated manual transmission (AMT) vehicles as an example, we can express the vehicle dynamics equation in a zero-load condition as follows:

$$
\frac{T_{t q} i_{g} i_{0} \eta_{T}}{r}=m_{z} g f_{z}+\frac{C_{D z} A}{21.15} u_{a}^{2}+\delta_{z} m_{z} \frac{d u}{d t} .
$$

The term on the left is the current vehicle driving force, $F_{t}$, where $T_{t q}$ is the actual engine output torque, $i_{g}$ is the current gearbox ratio, $i_{0}$ is the final drive gear ratio, $\eta_{T}$ is the transmission mechanical efficiency, and $r$ is the wheel rolling radius.

The first term on the right refers to the rolling resistance $F_{f z}$ on a flat road, where $m_{z}$ is the mass of a no-load vehicle, $g$ is the acceleration of gravity, and $f_{z}$ is the rolling resistance coefficient on standard road.

The second term is the aerodynamic resistance $F_{w z}$, where $C_{D z}$ is the aerodynamic resistance coefficient, $A$ is the frontal area, and $u_{a}$ is the vehicle speed in unit $\mathrm{km} / \mathrm{h}$.

The third item refers to accelerating resistance, $F_{j z}$, where $u$ is the vehicle speed in unit $\mathrm{m} / \mathrm{s}$ and $\delta_{z}$ is the correction coefficient of the rotating mass with no load calculated by the following equation:

$$
\delta_{z}=1+\frac{\sum I_{w}+I_{f} i_{g}{ }^{2} i_{0}{ }^{2} \eta_{T}}{m_{z} r^{2}}
$$

where $I_{w}$ is the wheel moment of inertia and $I_{f}$ is the flywheel moment of inertia.

Next, the terms of $\eta_{T} / r$ are moved to the right side of (2). The term on the left is the current gearbox output torque, $T_{t q}$, whereas the term on the right is the sum of all types of resistance torques in zero-load condition. Based on (1), the vehicle load can be re-redefined as follows:

$$
L_{\mathrm{Nm}}=T_{t q} i_{g} i_{0}-\left(m_{z} g f_{z}+\frac{C_{D z} A}{21.15} u_{a}^{2}+\delta_{z} m_{z} \frac{d u}{d t}\right) \frac{r}{\eta_{T}} .
$$

Equation (4) shows that the vehicle load $L_{\mathrm{Nm}}$ is a torquebased expression in Nm. It represents the torque difference between the gearbox output torque in the current driving condition and resistance torques in a zero-load condition when driving with the same vehicle speed and acceleration.

The reason for converting (1) is that the parameters $\eta_{T}$ and $r$ can be affected by the driving condition. Through conversion, $\eta_{T}$ and $r$ are included in the resistance torques, and the driving torque is calculated directly by the engine torque and ratio. The parameters of other resistance torques can be obtained through calibration in a zero-load driving condition.

2.3. Principle of Driving Condition Recognition Based on Vehicle Load. If we suppose that one or more parameters 
are changed in (4) (i.e., one or more constraint conditions in the zero-load condition are changed), then $L_{\mathrm{Nm}}$ varies accordingly and reflects these alternations. If we can obtain the vehicle load $L_{\mathrm{Nm}}$ accurately in real time, then the driving condition information can be recognized. Its recognition result can then be used in the vehicle control strategy.

The principle of vehicle load-based driving condition recognition method is just the calculation process of $L_{\mathrm{Nm}}$. Thus, the part of $\left(m_{z} g f_{z}+\left(C_{D z} A / 21.15\right) u_{a}{ }^{2}+\delta_{z} m_{z}(d u)\right.$ $d t))\left(r / \eta_{T}\right)$ can be seen as a vehicle reference model used to calculate the driving torque under a zero-load condition. $T_{t q} i_{g} i_{0}$ is obtained from the actual vehicle model used to calculate the current driving torque. The process of computing the generalized load requires the current gearbox output torque and resistance torques in a zero-load condition with the same vehicle speed and acceleration. The following chapter analyzes the effects of different driving conditions on recognition results and then provides detailed recognition steps.

\section{Influence Factor Analysis of Generalized Vehicle Load Recognition}

Given that the generalized vehicle load concept is defined in a zero-load condition, its recognition result in a zero-load condition should be investigated first before each influence factor in different driving conditions can be analyzed.

\subsection{Vehicle Load Recognition in a Zero-Load Condition.} Although a zero-load condition qualifies some driving behavior and loading, weather, and road conditions in vehicle system dynamics, each resistance torque in (4) remains under the influence of parameters such as the vehicle speed and correction coefficient of rotating mass. In particular, the driver behavior patterns of gear shift, acceleration, and deceleration can cause resistance changes accordingly. However, vehicle driving force should always be equal to the sum of all resistances based on the vehicle driving-resistance balance equation [16]. Therefore, the recognition result of (4) in a zero-load condition should theoretically always be maintained at 0 regardless of how a driver steps on the gas pedal (i.e., at any vehicle speed and acceleration). This condition is also the origin of the term zero-load condition.

\subsection{Grade Factor}

3.2.1. Uphill Condition. When changing from a flat and straight road in a zero-load condition into an uphill condition, the vehicle dynamics equation contains the grade resistance. The rolling resistance is also affected. At this point, the following equation is used:

$$
\begin{aligned}
& T_{t q} i_{g} i_{0}=\left(m_{z} g f_{z} \cos \theta+\frac{C_{D z} A}{21.15} u_{a}^{2}+\delta_{z} m_{z} \frac{d u}{d t}\right. \\
& \left.+m_{z} g \sin \theta\right) \frac{r}{\eta_{T}},
\end{aligned}
$$

where $\theta$ is the angle of grade.

Grade resistance exists during uphill driving. Given the balanced relationship between the vehicle driving force and external resistances, a driver is required to fully step on the gas pedal to counterbalance the grade resistance and reach the same vehicle speed and acceleration in a zero-load condition. Thus, the gearbox output torque is larger than that in a zeroload condition. By substituting (5) into (4), we obtain the load expression, $L_{i_{-} \text {up }}$, when driving uphill. Thus,

$$
L_{i_{\text {uup }}}=\left[m_{z} g f_{z}(\cos \theta-1)+m_{z} g \sin \theta\right] \frac{r}{\eta_{T}} \text {. }
$$

Equation (6) shows that the load recognition result contains two components of the rolling resistance torque and grade resistance torque. However, the rolling resistance has minimal effect on the load recognition result because the angle of grade is generally small.

3.2.2. Downhill Condition. During downhill driving, the grade resistance is in the same direction as the driving force and plays the role of an accelerating vehicle. At this point, the gearbox output torque is less than that under a zeroload condition with the same vehicle speed and acceleration. Therefore,

$$
\begin{aligned}
& T_{t q} i_{g} i_{0}=\left(m_{z} g f_{z} \cos \theta+\frac{C_{D z} A}{21.15} u_{a}^{2}+\delta_{z} m_{z} \frac{d u}{d t}\right. \\
& \left.-m_{z} g \sin \theta\right) \frac{r}{\eta_{T}} .
\end{aligned}
$$

The driver releases the throttle or simultaneously brakes to decelerate. The gearbox output torque then decreases. When not braking, we obtain the vehicle load expression of downhill, $L_{i_{-} \mathrm{dw}}$, by substituting (7) into (4). Thus,

$$
L_{i_{-} \mathrm{dw}}=\left[m_{z} g f_{z}(\cos \theta-1)-m_{z} g \sin \theta\right] \frac{r}{\eta_{T}} .
$$

Given $m_{z} g f_{z}(\cos \theta-1)<0$, the vehicle load while driving downhill is negative when not braking. Consider

$$
L_{i_{-} \mathrm{dw}}<0 .
$$

Given that the braking force originates from the brake system, the vehicle load recognition result is larger than (8) when braking and is incorrect. This study does not consider the braking condition.

Thus, the load recognition result is positive when driving uphill and negative when driving downhill, and its absolute value increases with the angle of grade.

3.3. Loading Mass Factor. In the case of increasing loading in a zero-load condition, the rolling and accelerating resistances affected by the loading mass in (4) increase synchronously. Given the force balance rule, the gearbox should output a higher torque to reach the same vehicle speed and acceleration in a zero-load condition. Thus, the load recognition result $L_{m}$ calculated from (4) increases correspondingly. Setting $m_{n}$ as the new vehicle mass with loading, we can express $L_{m}$ as follows:

$$
L_{m}=\left[\left(m_{n}-m_{z}\right) g f_{z}+\left(\delta_{n} m_{n}-\delta_{z} m_{z}\right) \frac{d u}{d t}\right] \frac{r}{\eta_{T}},
$$




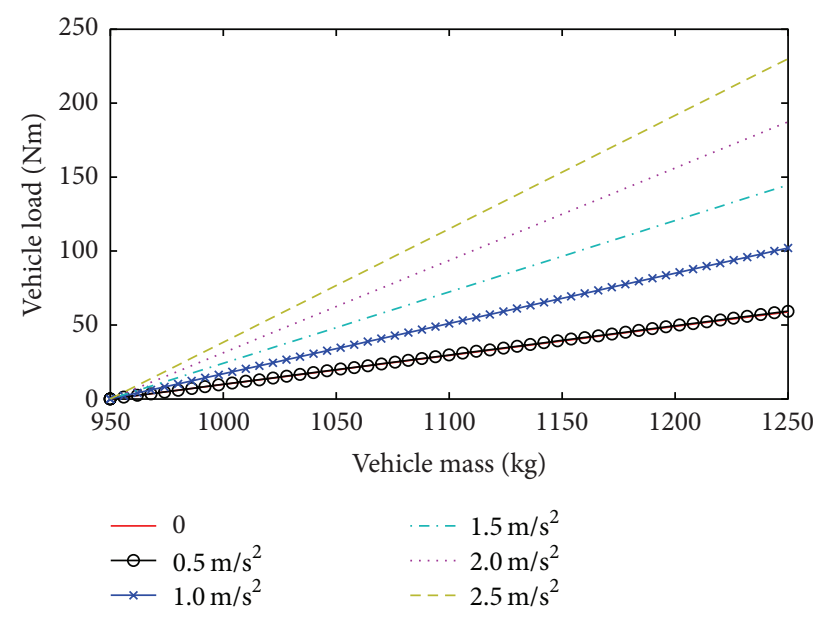

FIGURE 1: Effects of loading mass on load recognition result.

where $\delta_{n}$ is the correction coefficient of the rotating mass after the loading mass is increased.

Equation (3) shows that the correction coefficient of the rotating mass is also under the influence of the loading mass. Thus, (10) considers $\delta_{n}$.

Equation (10) shows that the vehicle load recognition result contains the two components of rolling resistance torque and acceleration resistance torque. The rolling resistance torque is almost constant, whereas the accelerating resistance torque is affected not only by the current loading mass but also by the vehicle longitude acceleration. Figure 1 shows the simulation result of the vehicle load recognition result of a minitype AMT vehicle when the loading mass increases from no load to full load at different acceleration values. The results indicate that the higher the acceleration value, the larger the load recognition result.

Vehicle acceleration is maintained in a low range and the rolling resistance torque component plays a significant role in most cases, but the effect is weak. Figure 1 shows that the curves at 0 and $0.5 \mathrm{~m} / \mathrm{s}^{2}$ are near. If the loading mass is large such as the case in a full load, or if acceleration is high, then the loading effect on load recognition is significant.

3.4. Rolling and Aerodynamic Resistance Factors. If the weather and road conditions in a zero-load condition change simultaneously or separately, then the aerodynamic resistance coefficient and rolling resistance coefficient change correspondingly. Using the same analysis process, we find that the load $L_{f}$ caused by road conditions and $L_{w}$ caused by weather conditions increase or decrease, respectively. Thus, we can obtain the following equation:

$$
L_{f}+L_{w}=\left[m_{z} g\left(f_{n}-f_{z}\right)+\frac{C_{D n} A-C_{D z} A}{21.15} u_{a}^{2}\right] \frac{r}{\eta_{T}},
$$

where $f_{n}$ is the current (now) rolling resistance coefficient and $C_{D n}$ is the current (now) aerodynamic resistance coefficient.

3.5. Braking Factor. As the braking force is an unconventional external resistance, the braking behavior is disallowed in a zero-load condition. However, braking occurs frequently during normal driving. At this point, the type-ground braking force takes effect, which is equivalent to the increasing external resistances. Thus, the load recognition result also increases as follows:

$$
F_{t}=F_{f}+F_{w}+F_{j}+F_{b}
$$

where $F_{b}$ is the braking force. The recognition result clearly does not reflect the real situations at this point. Therefore, the braking effect should be seriously considered. The following adaptive strategy can be used during the load recognition process to eliminate the braking effect. First, the braking signal should be monitored in real time. When braking is detected, load values before braking are maintained. After the brake is released, a counter is set up because the braking effect does not disappear immediately. The load recognition calculation continues only when it reaches a certain counting number.

3.6. Curve Factor. The overall resistances when driving in a curve also include the resistance caused by the turning operation. Thus, the load recognition result in a curve is larger than that in a straight line. If the curve condition can be detected by other algorithms, the load recognition should be preserved and its old value should be maintained until it is in a straight line again. Otherwise, the recognition results are inaccurate.

3.7. Slipping Factor. The vehicle speed calculated by the wheel or output shaft speeds is higher than normal during the slipping condition. It does not reflect the real situation and load recognition result because of its error. Therefore, this value is similar to that in the previous chapter; that is, the old value should be maintained if slipping can be detected by other algorithms. Otherwise, this recognition result is inaccurate.

3.8. Analyses of All Factors. Each resistance simultaneously changes in actual driving conditions, so the load recognition result is the sum of the effects of all resistances. This value can be expressed as follows:

$$
L_{\mathrm{Nm}}=L_{i}+L_{m}+L_{f}+L_{w} .
$$

Table 1 summarizes the influences of different factors on the recognition result. The grade has a generally stronger effect followed by the loading mass. However, the effects of the rolling resistance and aerodynamic resistance are relatively small.

\section{Recognition of Resistance Torques in Zero-Load Condition}

The key to load recognition is to keep the recognition result always zero in a zero-load condition regardless of the vehicle speed and acceleration. This value can be changed by calibrating the rolling resistance, aerodynamic resistance, and accelerating resistance to effectively identify the load value 
TABLE 1: Effects of different factors.

\begin{tabular}{lc}
\hline Factors & Effects on recognition result \\
\hline Grade & Strong, especially for large grade \\
\hline Loading mass & $\begin{array}{c}\text { Medium, especially for full load } \\
\text { or trailer condition }\end{array}$ \\
\hline Weather & Weak \\
\hline Road condition & Weak \\
\hline Braking & $\begin{array}{c}\text { Abnormal, it should preserve } \\
\text { recognition operation }\end{array}$ \\
\hline Turning & $\begin{array}{c}\text { Abnormal, it should preserve } \\
\text { recognition operation }\end{array}$ \\
\hline
\end{tabular}

when the grade, loading, aerodynamic resistance, and rolling resistance factors change in normal driving conditions. The resistances in (4) can be calculated through simulation according to the existing vehicle parameters, but these values are imprecise and cannot be used in actual application directly. Thus, further calibration work should be conducted under the direction of simulation results. Note that the load value cannot always be zero because of signal deviations in actual calibration but should be in a certain small range. The following chapters describe the detailed steps for calibration.

4.1. Calculation of Gearbox Output Torque. Before load recognition, the gearbox output torque $T_{t q}$ should be obtained first. It can generally be calculated as the product of the engine output torque and current ratio. Obtaining the current engine output torque can be performed in two approaches. The first approach is by obtaining the electronic control unit calculated engine torque through the CAN bus. The second approach is by querying the engine performance MAP $T_{t q}\left(\alpha, n_{e}\right)$ based on the throttle opening and engine speed. Torque-based engine control strategy is adopted by the target vehicle, which can dynamically calculate the engine indicated torque and engine losses in various conditions. And the mathematical model between engine control parameters and engine output torque is determined by numerous experimental results and calibration. With the development of electronic control technology, the real-time engine output torque calculated by ECU is sufficiently accurate. Therefore, the first approach is recommended. If the second approach is used, it can result in load recognition errors because the engine performance curves are obtained in a steady operation condition and deviates from that in an actual operation condition.

Before calculating the gearbox output torque, the filtration operation for the raw engine output torque should be used to filter out dithering components. Otherwise, it will easily lead to fluctuations in the load recognition result. The first-order low-pass digital filter is used in this study, which can be expressed as follows:

$$
y_{n}=a x_{n}+(1-a) y_{n-1} \text {, }
$$

where $x_{n}$ is the new sample value, $y_{n-1}$ is the last sample value, $a$ is the filter coefficient, and $y_{n}$ is the filtered result. Figure 2 shows the curves before and after filtering.
TABLE 2: Calibration of road resistance torque.

\begin{tabular}{lllllll}
\hline Vehicle speed Km/h & 20 & 40 & 60 & 80 & 100 & 120 \\
\hline Road resistance Nm & 35 & 50 & 67 & 95 & 150 & 170 \\
\hline
\end{tabular}

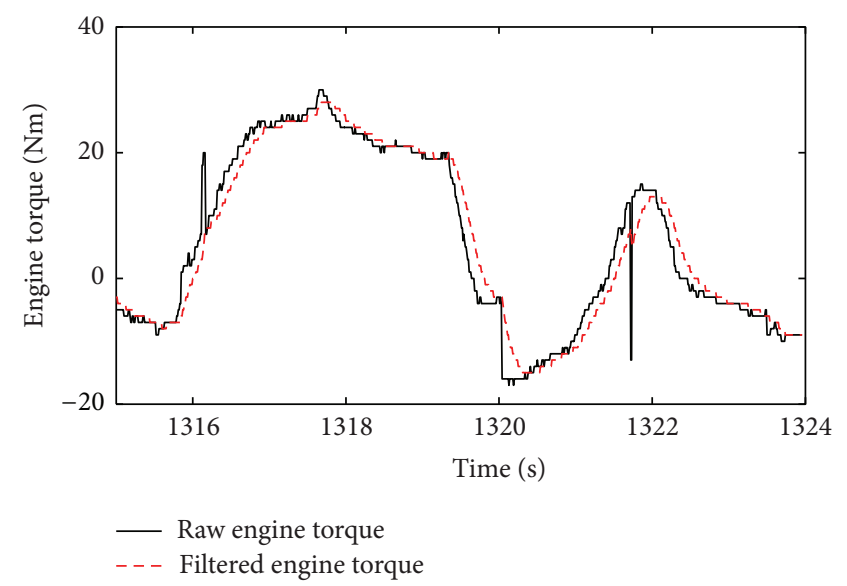

FIGURE 2: Filtration of the engine torque.

4.2. Calibration of Aerodynamic and Rolling Resistance Torques. The aerodynamic and rolling resistance torques, that is, road resistance torque in a zero-load condition, should then be calibrated.

All resistances act on the gearbox output shaft at the same time during vehicle driving. Therefore, we should ask drivers to drive the vehicle at a constant speed to calibrate road resistance torque successfully. This condition means that vehicle acceleration is zero and the accelerating resistance torque is eliminated. Thus, we can focus only on the calibration of road resistance torque. At this point, the vehicle load is calculated as follows on the basis of (4):

$$
L_{\mathrm{Nm}}=T_{t q}-\left(m_{z} g f_{z}+\frac{C_{D z} A}{21.15} u_{a}^{2}\right) \frac{r}{\eta_{T}} .
$$

The purpose of calibration in this step is to maintain the vehicle load value in an acceptable small range. The rolling resistance coefficient is mainly affected by the road condition, vehicle speed, and tire parameters [16]. However, the road condition and tire parameters are qualified in a zero-load condition, so the coefficient $f_{z}$ can be used as a vehicle speed function. The aerodynamic resistance torque in (15) is also a vehicle speed function. Therefore, the road resistance torque is also a vehicle speed function in the preceding analysis. Thus, the main task of this step is to calibrate the road resistance torque under different vehicle speeds to set the vehicle load value to zero in a zero-load condition.

Given different inevitable errors, the vehicle load value cannot always be zero in the actual calibration process but should be in an acceptable small range. Table 2 shows the calibration results of the road resistance torque for an AMT car.

4.3. Calibration of Accelerating Resistance Torque. After obtaining the rolling and aerodynamic resistance, only the accelerating resistance torque remains in (4). Thus, 
TABLE 3: Calibration of acceleration resistance.

\begin{tabular}{cccccc}
\hline$i_{g}$ & 1 & 2 & 3 & 4 & 5 \\
\hline$\frac{\delta_{z} m_{z} r}{\eta_{T}}$ & 352 & 303 & 290 & 287 & 285 \\
\hline
\end{tabular}

this step calibrates the accelerating resistance torque, $\delta_{z} m_{z}\left(r / \eta_{T}\right)(d u / d t)$, under different accelerations in a zero-load condition. The goal is also to set the load in an acceptable small range.

First, vehicle acceleration is required before calibration. Given that the vehicle speed sensor is a common device in modern automobiles, acceleration can be directly obtained from it, but its accuracy is sometimes not sufficiently high. If the gearbox output shaft speed sensor is installed, acceleration can be calculated because its signal is more accurate as used in this study. In addition, acceleration signals must be filtered; otherwise, it becomes unstable and affects the recognition result. A first-order low-pass digital filter is used in this study.

Equation (3) shows that the correction coefficient of the rotating mass $\delta_{z}$ is affected by the gearbox ratio in a zeroload condition. Therefore, the main task of this step is to calibrate the term of $\delta_{z} m_{z}\left(r / \eta_{T}\right)$ under different ratios or gears. Table 3 lists the calibration results of accelerating the resistance torque of an AMT car.

Thus, the load value in a normal condition can be recognized and can reflect the actual driving condition after each resistance torque in a zero-load condition is determined.

\section{Adaptive Gearshift Strategy Based on Generalized Vehicle Load Identification}

Generalized vehicle load synthetically reflects the sum of the entire driving resistance in general driving environment. The basic idea of gearshift strategy based on generalized load recognition is according to recognition results to select the gear suitable for the current general driving environment, which equals making a choice from a bunch of optimal twoparameter shift schedules under different driving resistance.

5.1. Theoretical Derivation. Adaptive gearshift strategy based on generalized load has close relationship with dynamic three-parameter shift schedules which can resolve the problem of application difficulty. This method can be convenient and easy to implement for real vehicle control. In this paper, the principle of this adaptive gearshift strategy is deduced based on three-parameter shift schedule.

According to the definition of generalized load,

$$
F_{j z}=F_{t}-F_{f z}-F_{w z}-L_{N}
$$

Therefore, the formula of acceleration in general driving environment is available; namely,

$$
\frac{d u}{d t}=\frac{1}{\delta_{z} m_{z}}\left(\frac{T_{e} i_{g} i_{0} \eta_{T}}{r}-m_{z} g f_{z}-\frac{C_{D z} A}{21.15} u_{a}^{2}-L_{N}\right) .
$$

The parameters in formula [17] can be got through the resistance torque recognition in zero-load condition. Acceleration in general driving environment can be calculated utilizing current engine torque, rolling and air resistance in zero-load condition, and generalized vehicle load.

Engine torque is a function of vehicle speed and throttle opening [17]; namely,

$$
T_{t q}=f\left(\alpha, u_{a}\right)
$$

And rolling and air resistance in zero-load condition are the function of vehicle speed. One has

$$
F_{f z}+F_{w z}=f\left(u_{a}\right) .
$$

Therefore, acceleration can be expressed with the throttle opening, vehicle speed, and generalized load. Consider

$$
\frac{d u}{d t}=f\left(\alpha, u_{a}, L_{N}\right) .
$$

Three-parameter shift schedule is represented by acceleration, throttle, and vehicle speed, [17] namely, $\left(d u / d t, \alpha, u_{a}\right)$. Equation (20) shows that acceleration is determined by throttle, vehicle speed, and generalized vehicle load. Therefore, three-parameter shift schedule can be converted into the new form based on generalized load, vehicle speed, and throttle. At this time, shift schedule is based on generalized load called adaptive gearshift strategy, which can be expressed as follows: $\left(L_{N}, \alpha, u_{a}\right)$.

Through the above analysis, adaptive gearshift strategy based on generalized load essentially consistent with threeparameter shift schedule can be adaptive to general driving environment.

5.2. Gearshift Strategy Based on Generalized Vehicle Load. According to the definition, generalized load can recognize general driving environment. Different generalized load stands for different working conditions in general driving environment; therefore, gearshift strategy based on generalized load does not need to individually develop appropriate strategies for working conditions such as loading and climbing,. Corresponding shift lines can be obtained by using an interpolation method according to generalized load recognized result to meet the dynamic needs.

Interpolation between shift schedule curve on flat and largest gradient based on recognition results can realize adaptive gearshift decision under general driving environment. For the convenience of application, normalization processing is used to transform generalized load results into a normalized $L$ in $[-100,100]$, where -100 stands for the normalized generalized load in the maximum downhill condition and 100 stands for the normalized generalized load in the maximum uphill condition. The interpolation equation is shown as follows:

$$
v_{\text {load }}=v_{o}+\frac{L}{100}\left(v_{\text {hill }}-v_{o}\right) .
$$

In the equation, $v_{\text {load }}$ stands for a shift line based on generalized vehicle load, $v_{o}$ stands for shift lines on flat road, and $v_{\text {hill }}$ stands for shift lines on the maximum slope road. 
TABLE 4: Contrastive analysis of different gearshift schedules.

\begin{tabular}{|c|c|c|c|c|c|c|c|}
\hline \multirow[t]{2}{*}{ Working condition } & \multicolumn{3}{|c|}{$\begin{array}{l}\text { Adaptive gearshift strategy } \\
\text { based on generalized load }\end{array}$} & \multicolumn{3}{|c|}{$\begin{array}{c}\text { Dynamic three-parameter } \\
\text { shift schedule }\end{array}$} & \multirow[t]{2}{*}{ Formulation principle } \\
\hline & $L_{N}$ & $\alpha$ & $u_{a}$ & $d u / d t$ & $\alpha$ & $u_{a}$ & \\
\hline \multirow{6}{*}{ Flat road } & 0 & 10 & 20.3 & 0.616 & 10 & 20.3 & Boundary point, maintain the original gear \\
\hline & 0 & 20 & 20.3 & 0.616 & 20 & 20.3 & Acceleration is equal \\
\hline & 0 & 30 & 21.6 & 0.815 & 30 & 21.6 & Acceleration is equal \\
\hline & 0 & 40 & 25.6 & 1.188 & 40 & 25.6 & Acceleration is equal \\
\hline & 0 & 60 & 32.1 & 2.202 & 60 & 32.1 & Boundary point, upshift to limit speed \\
\hline & $\vdots$ & $\vdots$ & $\vdots$ & $\vdots$ & $\vdots$ & $\vdots$ & $\vdots$ \\
\hline \multirow{6}{*}{ Slope road $(5.2 \%)$} & 147 & 10 & 23.6 & 0.122 & 10 & 23.6 & Boundary point, maintain the original gear \\
\hline & 147 & 20 & 23.6 & 0.122 & 20 & 23.6 & Acceleration is equal \\
\hline & 147 & 30 & 24.2 & 0.261 & 30 & 24.2 & Acceleration is equal \\
\hline & 147 & 40 & 28 & 0.723 & 40 & 28 & Acceleration is equal \\
\hline & 147 & 60 & 35.8 & 1.804 & 60 & 35.8 & Boundary point, upshift to limit speed \\
\hline & $\vdots$ & $\vdots$ & $\vdots$ & $\vdots$ & $\vdots$ & $\vdots$ & $\vdots$ \\
\hline \multirow{6}{*}{ Slope road $(14 \%)$} & 389 & 10 & 27.9 & 0.064 & 10 & 27.9 & Boundary point, maintain the original gear \\
\hline & 389 & 20 & 27.9 & 0.064 & 20 & 27.9 & Boundary point, maintain the original gear \\
\hline & 389 & 30 & 28.5 & 0.064 & 30 & 28.5 & Boundary point, maintain the original gear \\
\hline & 389 & 40 & 33.1 & 0.064 & 40 & 33.1 & Boundary point, upshift to limit speed \\
\hline & 389 & 60 & 42.1 & 1.146 & 60 & 42.1 & Boundary point, upshift to limit speed \\
\hline & $\vdots$ & $\vdots$ & $\vdots$ & $\vdots$ & $\vdots$ & : & $\vdots$ \\
\hline
\end{tabular}

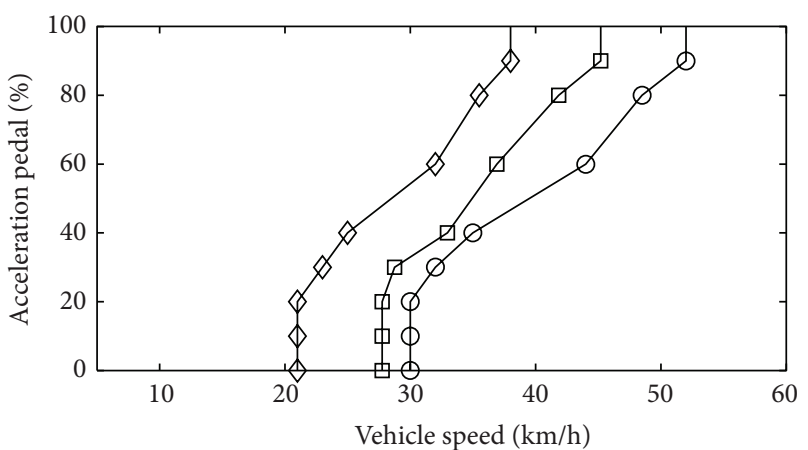

$$
\begin{aligned}
& \diamond \text { Flat 1-2 } \\
& \ominus \text { Uphill 1-2 } \\
& \square \text { Downhill 1-2 }
\end{aligned}
$$

FIGURE 3: Shift lines based on generalized load.

The shift line on the maximum downhill designed for a testing AMT vehicle is shown in Figure 3. The shift lines on a flat road and the maximum uphill slope road are also shown in Figure 3. The interpolation results based on generalized load are closer to the shift line on flat road when the slope is lower and when the slope is higher the results are closer to the shift line on a downhill which has a better effect of the engine auxiliary braking.

5.3. Characteristic Analysis of the Shift Lines Based on Generalized Load. Adaptive gearshift strategy based on generalized load and dynamic three-parameter shift schedule designed for a testing AMT vehicle are shown in Table 4. In order to illustrate clearly, the working conditions are set as flat road (generalized load is 0), common slope (with 5.2\% slope and generalized load is $147 \mathrm{~N}$ ), and the maximum slope at 2-shift gear (with 14\% slope and generalized load is $389 \mathrm{~N}$ ).

The italicized parts in the table show that throttle angles and vehicle speeds are the same in both shift lines. Through the analysis of the data in the table, we can get the following:

(1) Acceleration at shift time on slope road is obviously lower than that on flat road with the same throttle angle, which proves that generalized load reflects the external resistances. Therefore gearshift strategy based on generalized load can be adaptive to the general driving environment.

(2) Generalized load remains unchanged under the same working condition, and it can be expressed by a cluster of shift lines under different generalized load. Therefore, gearshift strategy based on generalized load has a more concise expression. And it needs to consider different accelerations for dynamic threeparameter shift schedule which means it needs to be represented with tridimensional curved surface.

(3) By observing the data in Table 4, the shift speed on $5.2 \%$ slope road is between those on flat road and $14 \%$ slope road with the same throttle angle. It can be taken as an example that the shift speeds are $21.6 \mathrm{~km} / \mathrm{h}$, $24.2 \mathrm{~km} / \mathrm{h}$, and $28.5 \mathrm{~km} / \mathrm{h}$ on flat road, common slope road, and maximum slope road with $30 \%$ throttle opening. It means that the current shift point can be obtained by using interpolations between the shift 

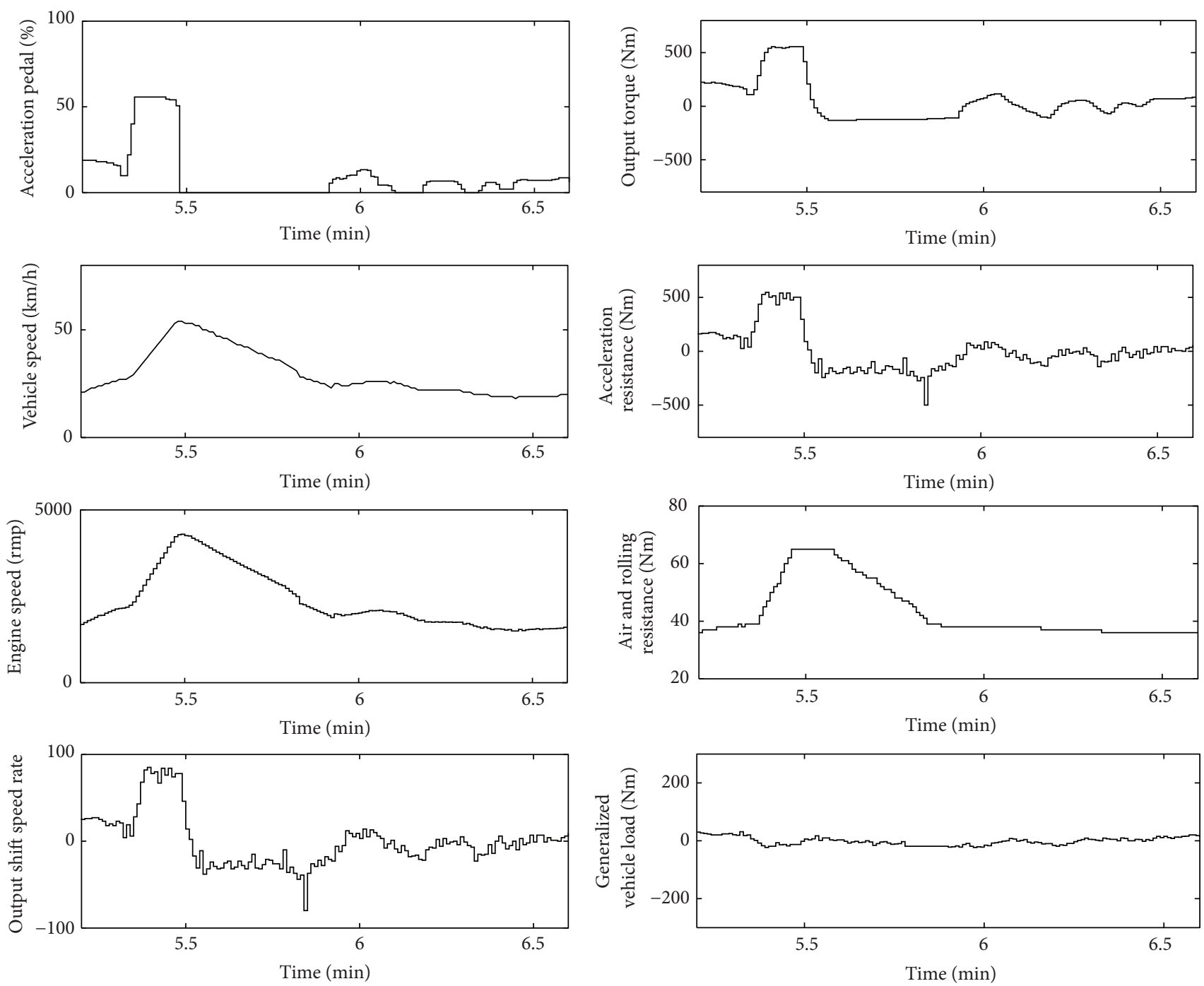

FIGURE 4: Generalized vehicle load recognition in zero-load condition.

point on flat road and maximum slope road according to the slope recognized. It proves the rationality of the interpolation method.

The above analyses show the relationship between gearshift strategy based on generalized load and dynamic three-parameter shift schedule. They all have adaptive capacity to the general driving environment. In addition, it has a more concise expression and is easy to be used in an actual vehicle control.

\section{Test and Analysis}

The vehicle load recognition algorithm has been validated in an AMT car. Experiments required signals, such as the engine torque, vehicle speed, and current gear obtained from CAN bus. Considering the fact that the acceleration signal is not easy to get, it is replaced with output shaft speed rate calculated by the output shaft speed per $300 \mathrm{~ms}$. The self-development TCU integrated with 16-bit microcontroller
ST10F276Z5 is adopted. It has enough arithmetic speed to meet real-time computing needs for the proposed method. Although filter processing of engine torque has been conducted before generalized vehicle load identification, the identification results will still have certain fluctuation. In order to get stable and smooth vehicle load data which is convenient for adaptive gearshift decision, the first-order low-pass filtering algorithm is adopted to process results.

6.1. Verification of Resistance Torque Recognition in Zero-Load Condition. Figure 4 shows calculation results of generalized vehicle load in zero-load condition after recognition of resistance torque and relevant parameters. Acceleration pedal is stepped on acutely and then released rapidly. It is observed that the air, rolling, and acceleration resistance torque are changing as the change of speed and acceleration. And generalized vehicle load always stays near zero. Therefore, vehicle load under actual working conditions can be got after resistance torque recognition in zero-load condition. 

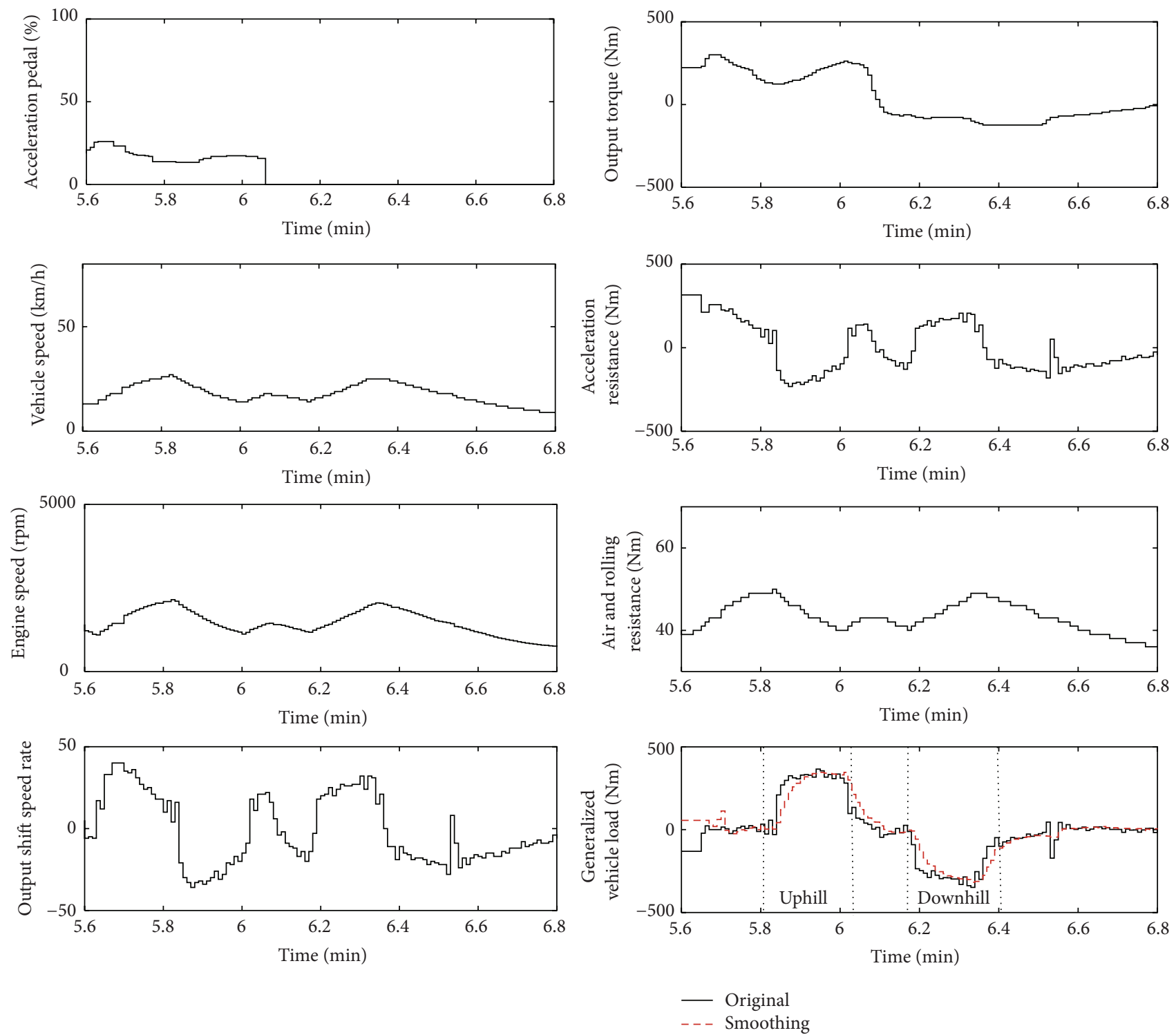

FIGURE 5: Vehicle load recognition result.

6.2. Verification of Vehicle Load Identification. Figure 5 shows the vehicle load recognition results when driving uphill with gradient $11 \%$ at the start, then 14 -meter flat road, and downhill with gradient $11 \%$ at the end. At beginning of uphill, the vehicle body sloped upward gradually, and vehicle load recognition result increased with the increasing of gearbox output torque; then when all wheels were on the slope totally, load value stabilized at about $280 \sim 320 \mathrm{Nm}$; when driving on flat road, the vehicle body laid flat gradually and load value decreased to about $0 \pm 15 \mathrm{Nm}$, which agreed well with the normal flat road. But as this road was short, the vehicle went downhill soon; at the beginning of downhill, the vehicle body sloped downward gradually. At this moment, the driver released the gas pedal with no braking, and the engine braking effect worked strongly. So load value decreased with the decreasing of gearbox output torque; finally, when all wheels were on the slope totally, load value stabilized at around $-340 \sim-320 \mathrm{Nm}$. We can see the disturbance of load value when downhill, which was caused by the uneven road surface. This has proved that the grade was recognized effectively.

6.3. Verification of Road Gradient Reconstruction. The above test is conducted specifically for slope road. In the process of testing other factors including load and wind speed are ignored. Therefore, the road gradient can be calculated based on generalized load identification results. Comparing with the actual gradient, generalized load identification algorithms can be verified.

According to (6),

$$
f_{z} \cos \theta+\sin \theta=\frac{\eta_{T} L_{i}}{m_{z} g r}+f_{z} .
$$




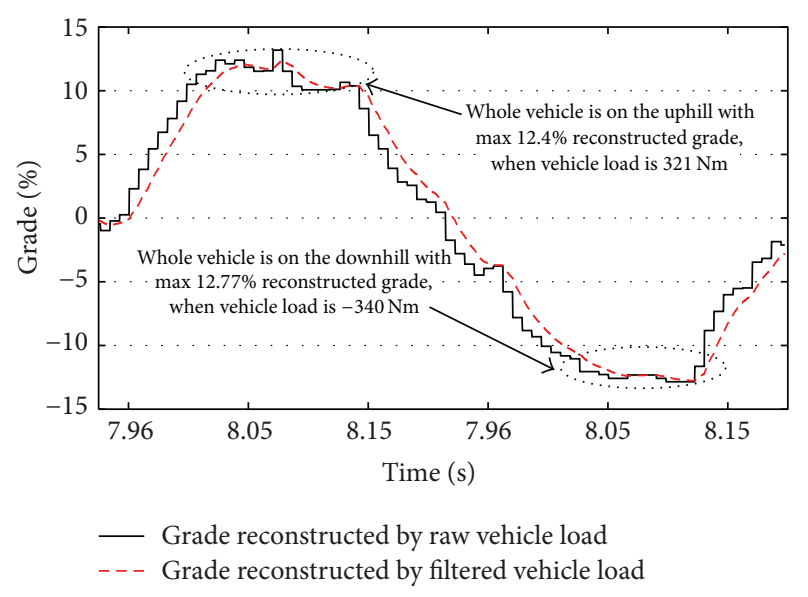

FIGURE 6: Result of grade reconstruction verification.

By trigonometric function auxiliary angle equation,

$$
f_{z} \cos \theta+\sin \theta=\sqrt{f_{z}^{2}+1} \cdot \sin \left(\theta+\arctan f_{z}\right) \text {. }
$$

Therefore, road gradient can be calculated as follows:

$$
\begin{aligned}
\theta= & \arcsin \left(\frac{1}{\sqrt{f_{z}^{2}+1}}\left(\frac{\eta_{T} L_{i}}{m_{z} g^{r}}+f_{z}\right)\right) \\
& -\arctan \left(f_{z}\right) .
\end{aligned}
$$

Figure 6 shows calculated road gradient using original and filtered generalized vehicle load. The actual gradient is $11 \%$. As can be seen from the figure, when four wheels are completely on uphill, calculated road gradient keeps in the range from $10.3 \%$ to $12.4 \%$, while on downhill, it keeps in the range from $11 \%$ to $12.77 \%$.

Engine torque and calibration data have a great influence on generalized load identification result. From the engineering perspective, the more accurate engine torque data and more precise calibration process, the more accurate generalized vehicle load identification results.

6.4. Comprehensive Road Test. Comprehensive road tests mainly include flat and slope road, which are common operating conditions during actual driving situation. According to the results, the adaptability of the proposed gear decision method for general driving situation is verified.

6.4.1. Comprehensive Flat Road Test. Figure 7 shows the results of flat road test in urban drive condition, during which quick and slow release and deep and light step on the acceleration pedal occurred. Generalized vehicle load, accelerator pedal, economical and sport gearshift line, adaptive gearshift line, and target gear are shown in Figure 7. These gearshift lines are the vehicle speed curves of gearshift strategy. In subplot 3, the economical shift line is the optimal economic gearshift strategy, and the sport shift line is the optimal performance gearshift strategy. They are got by theoretical calculation based on the target vehicle and used
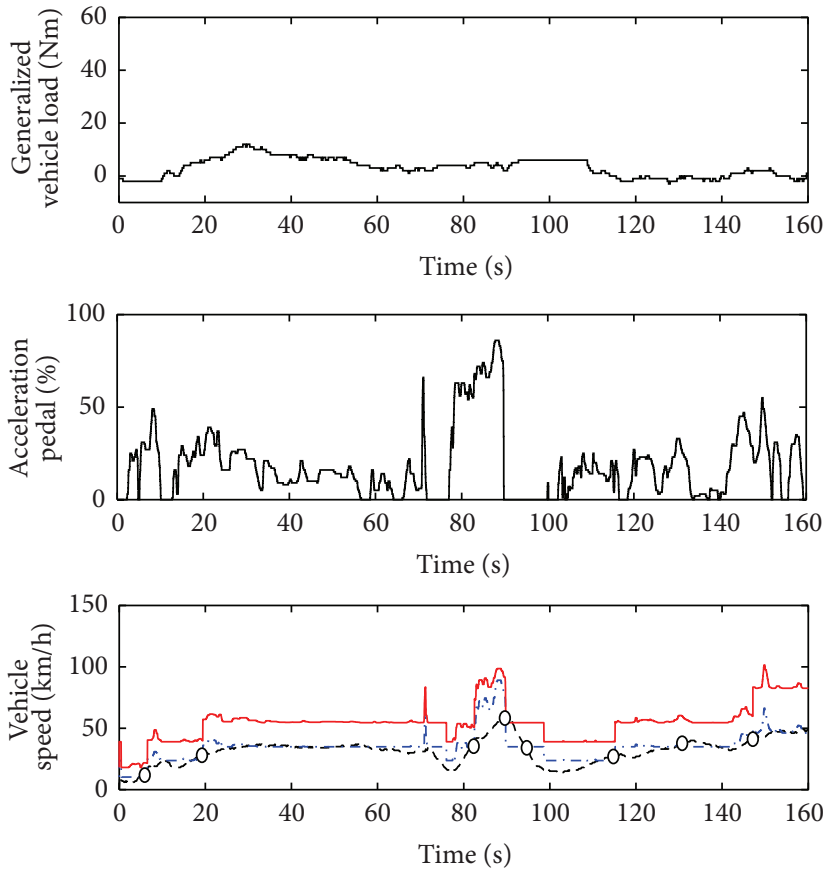

$$
\begin{array}{lll}
- \text { - }- \text { Vehicle speed } & & \text { Sport } \\
\text {-.- Economical } & 0 & \text { Basic shift point }
\end{array}
$$
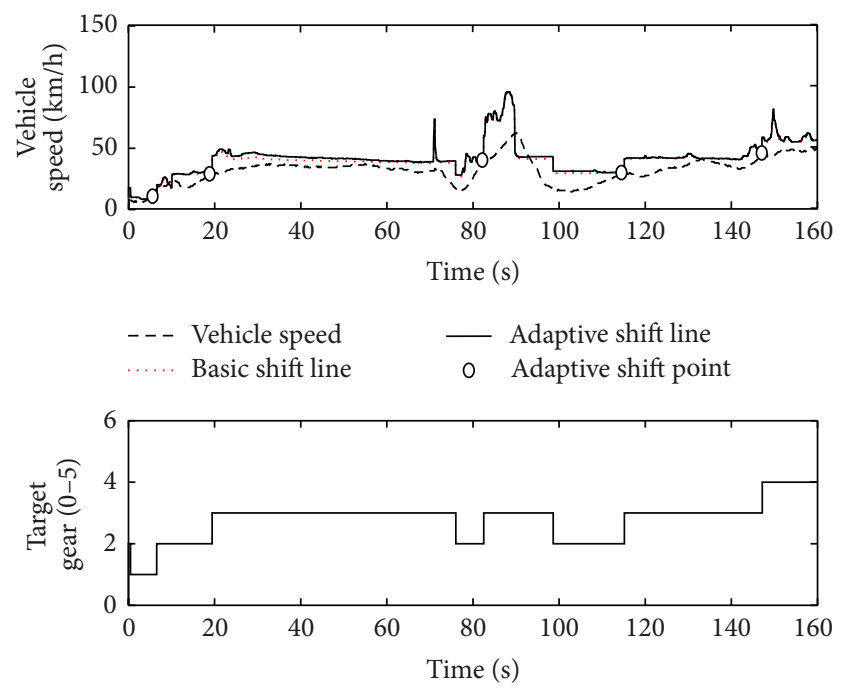

FIGURE 7: Comprehensive flat road test results.

for comparison with the proposed method. In subplot 4, basic shift line is the current production gearshift strategy used for the target vehicle, which have been optimized over many years based on experience using the target vehicle. The figure shows the following:

(1) Generalized vehicle load identification results change near 0 while driving on flat road. And adaptive shift line is basically identical with original shift line calibrated on flat road. There are a few cross points between the economical shift line and the velocity line. If gearshift decision is only conducted based on the economical shift line, the busy-shift problem will appear. 
(2) When accelerator pedal or its change rate is large, adaptive shift line will be closer to the sport shift line. At this time, the adaptive gear decision strategy is mainly tending to exert the vehicle dynamic performance. When it is small, adaptive shift line will be closer to the economical shift line. At this time, the gear decision strategy is tending to satisfy drivers' dynamic and economic requirement.

Therefore, the proposed method like the production shift strategy can ensure the optimal performance of the vehicle on flat road.

6.4.2. Comprehensive Slop Road Test. Before the comprehensive slope road test, it is conducted that the slope road test is based on conventional two-parameter shift schedule. If the shift schedule calibrated on the flat road is used, the busy-shift problem will appear on the slope road during the test process. This problem is particularly prominent on a long slope road. If the shift point is delayed artificially, the cycle shift phenomenon on the slope road will be eliminated. However, it will lead to deterioration of the economy, comfort, and shift noise.

Figure 8 shows the test data curves on the slope road near the suburb, where the adaptive gear decision method is adopted on the test vehicle. The uphill shift line is the maximum grade gearshift strategy, which is got by theoretical calculation based on the target vehicle. When the test is conducted, the driver changes the throttle to verify whether the busy-shift problem appears and study the process of the gear decision. The figure shows the following:

(1) When the generalized load increases on the slope road, the difference between the shift line on the flat road and the shift line based on the generalized load is great. This reflects that the process of gear decision can adapt to the driving situation on the slope road.

(2) The duration of the slope road test is $0-40 \mathrm{~s}$. There are a few cross points between the shift line on the flat road and the velocity line. If the shift line on the flat road is used to decide the gear, this will lead to busy-shift. After the correction of shift line based on the generalized vehicle load, the busy-shift problem does not appear. There is a cross point between the final shift line and the velocity line, which is the result of adaptive gearshift strategy.

(3) If the generalized load is great, the shift schedule will be closer to the sport shift schedule. At this time, the gear decision strategy is tending to exert the vehicle dynamic. While the generalized load is small, the shift schedule will be closer to the economical shift schedule. At this time, the gear decision strategy is tending to satisfy drivers' dynamic and economic requirement.

According to the test results, the gear correction strategy based on the generalized load identification can avoid busy-shift problem on the slope road, satisfy dynamic requirement in different driving situations, and be adaptive
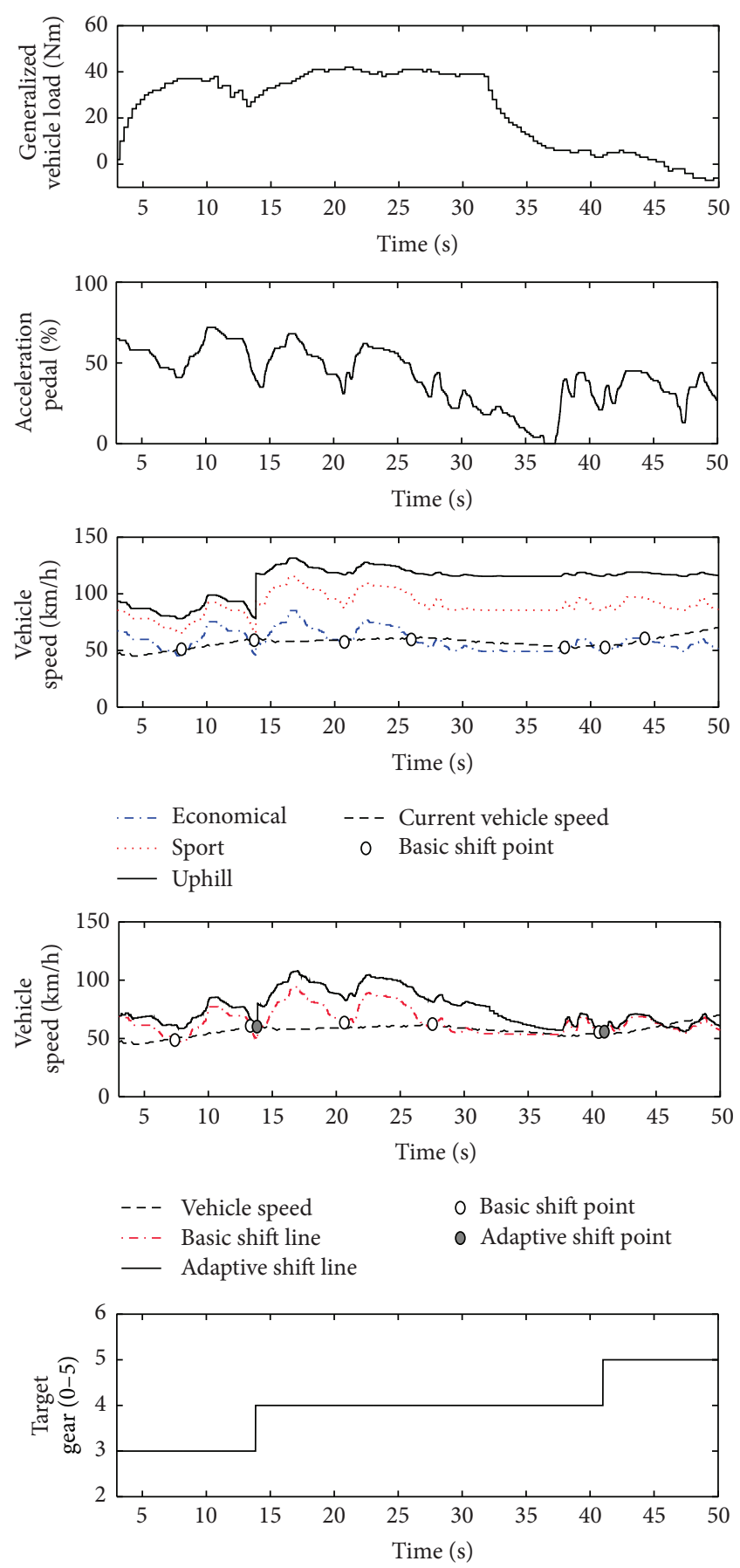

FIgURE 8: Comprehensive slope road test results.

to general driving situation. The slope road test results can also verify the adaptability in the situation of heavy duty, great wind drag, and rolling resistance, because the generalized load reflects vehicle resistance of general driving situation.

\section{Conclusions}

In this paper, the traditional load concept was extended to the generalized vehicle load, which reflects the external environment demand of vehicle power. Liner interpolation 
method between shift schedule curves on flat and on maximum gradient based on the recognition results is taken to produce adaptive gearshift decision under general driving environment. Compared with dynamic three-parameter shift schedule, it has concise expression instead of tridimensional curved surface and better real-time performance. And it reduces demands of memory space. This method requires only vehicle common sensors and does not need any other equipment. Vehicle test results verify the feasibility and validity of this method to improve adaptive ability of automatic transmission vehicles driving on general environment. At the same time, it can meet the power performance demand of vehicle driving environment and solve busy-shift problem on the slope road.

Vehicle driving environments vary widely; not only pavement type is complex, but also traffic flow condition under the same pavement type is always different. Different driving environment has different requirements for vehicle. This paper mainly studies the unified resistance identification method under general driving environment. But vehicle will actually face more complex environment. The study of special driving environment including curve, the low adhesion road (snow, muddy, and slippery road), and bumpy road will be taken as the next step to continually improve automatic transmission vehicle adaptability to the complicated driving environment.

\section{Conflict of Interests}

The authors declare that there is no conflict of interests regarding the publication of this paper.

\section{Acknowledgments}

This work was supported by International S\&T Cooperation Program of China (no. 2014DFA71790), the Key Scientific and Technological Project of Jilin Province (no. 20130204023GX), Doctoral Fund of the Ministry of Education of China (no. 20120061110027), "985 Project Automotive Engineering” of Jilin University, Program for Chang Jiang Scholars and Innovative Research Team in University (no. IRT1017), and Chinese High Technology Research Development Program (no. 2012AA111712).

\section{References}

[1] W. Yuhai, D. Ruixian, W. Song, and Z. Xuguang, "Real-time road slope recognition algorithm for heavy truck based on SAE J1939 protocol," Automotive Engineering, vol. 32, no. 7, pp. 640-647, 2010.

[2] J. Hui and G. Anlin, "A new vehicle intelligent shift system," Advances in Mechanical Engineering, vol. 2013, Article ID 303691, 2013.

[3] H. Jin, A. Ge, Y. Lei, and C. Zong, "Study on automobile automatic shift system based on driving environment recognition," Journal of Mechanical Engineering, vol. 38, no. 5, pp. 56-60, 2002.

[4] H. Ohnishi, J. Ishii, M. Kayano, and H. Katayama, "A study on road slope estimation for automatic transmission control," JSAE Review, vol. 21, no. 2, pp. 235-240, 2000.
[5] K. Jo, J. Kim, and M. Sunwoo, "Real-time road-slope estimation based on integration of onboard sensors with GPS using an IMMPDA filter," IEEE Transactions on Intelligent Transportation Systems, vol. 14, no. 4, pp. 1718-1732, 2013.

[6] I. Kim, H. Kim, J. Bang, and K. Huh, "Development of estimation algorithms for vehicle's mass and road grade," International Journal of Automotive Technology, vol. 14, no. 6, pp. 889-895, 2013.

[7] A. Vahidi, A. Stefanopoulou, and H. Peng, "Recursive least squares with forgetting for online estimation of vehicle mass and road grade: theory and experiments," Vehicle System Dynamics, vol. 43, no. 1, pp. 31-55, 2005.

[8] J. Shi, T. Lu, X. Li, and J. Zhang, "Self-adaptive slope gearshift strategy for automatic Transm ission vehicles," Journal of Agricultural Machinery, vol. 42, no. 4, pp. 1-7, 2011.

[9] K. Hayashi, Y. Shimizu, Y. Dote, A. Takayama, and A. Hirako, "Neuro fuzzy transmission control for automobile with variable loads," IEEE Transactions on Control Systems Technology, vol. 3, no. 1, pp. 49-53, 1995.

[10] A. Bastian, "Fuzzy logic in automatic transmission control," Vehicle System Dynamics, vol. 24, no. 4-5, pp. 389-400, 1995.

[11] S. Sakaguchi, I. Sakai, and T. Haga, "Application of fuzzy logic to shift scheduling method for automatic transmission," in Proceedings of the 2nd IEEE International Conference on Fuzzy Systems, pp. 52-58, April 1993.

[12] L. Qiao, M. Sato, and H. Takeda, "Learning algorithm of environmental recognition in driving vehicle," IEEE Transactions on Systems, Man and Cybernetics, vol. 25, no. 6, pp. 917-925, 1995.

[13] Y. H. Yamaguchi, H. Narita, H. Takahashi, and Y. Katou, "Automatic transmission shift schedule control using fuzzy logic," SAE Technical Paper 930674, 1993.

[14] K. Hebbale, D. Kim, C. J. Lee et al., "Smart shift adaptive schedule for automatic transmissions," Tech. Rep. F2008-06028, FISTA, 2008.

[15] S. Bai, K. V. Hebbale, and C. J. Lee, "Automatic transmission shift point control system and method of use," US Patent, Pub. no.: $\mathrm{WO} / 2008 / 014073$

[16] Z. Yu, Automobile Theory, Machinery Industry Press, Beijing, China, 2009.

[17] A. L. Ge, Vehicle Automatic Transmission Theory and Design, Machinery Industry Press, Beijing, China, 1993. 


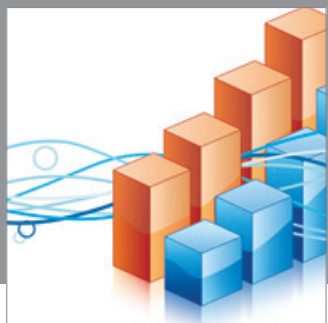

Advances in

Operations Research

mansans

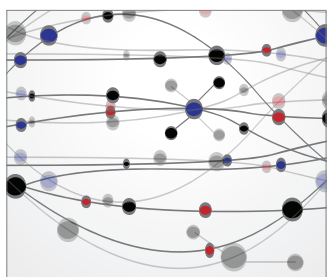

The Scientific World Journal
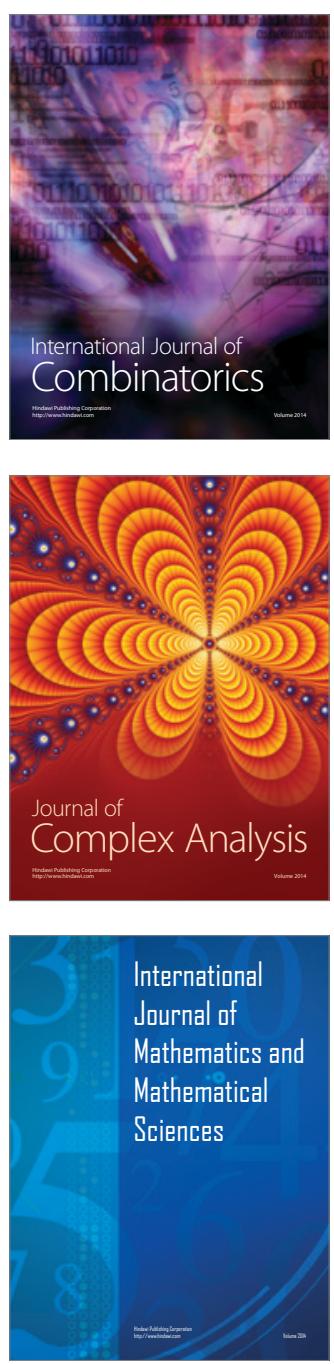
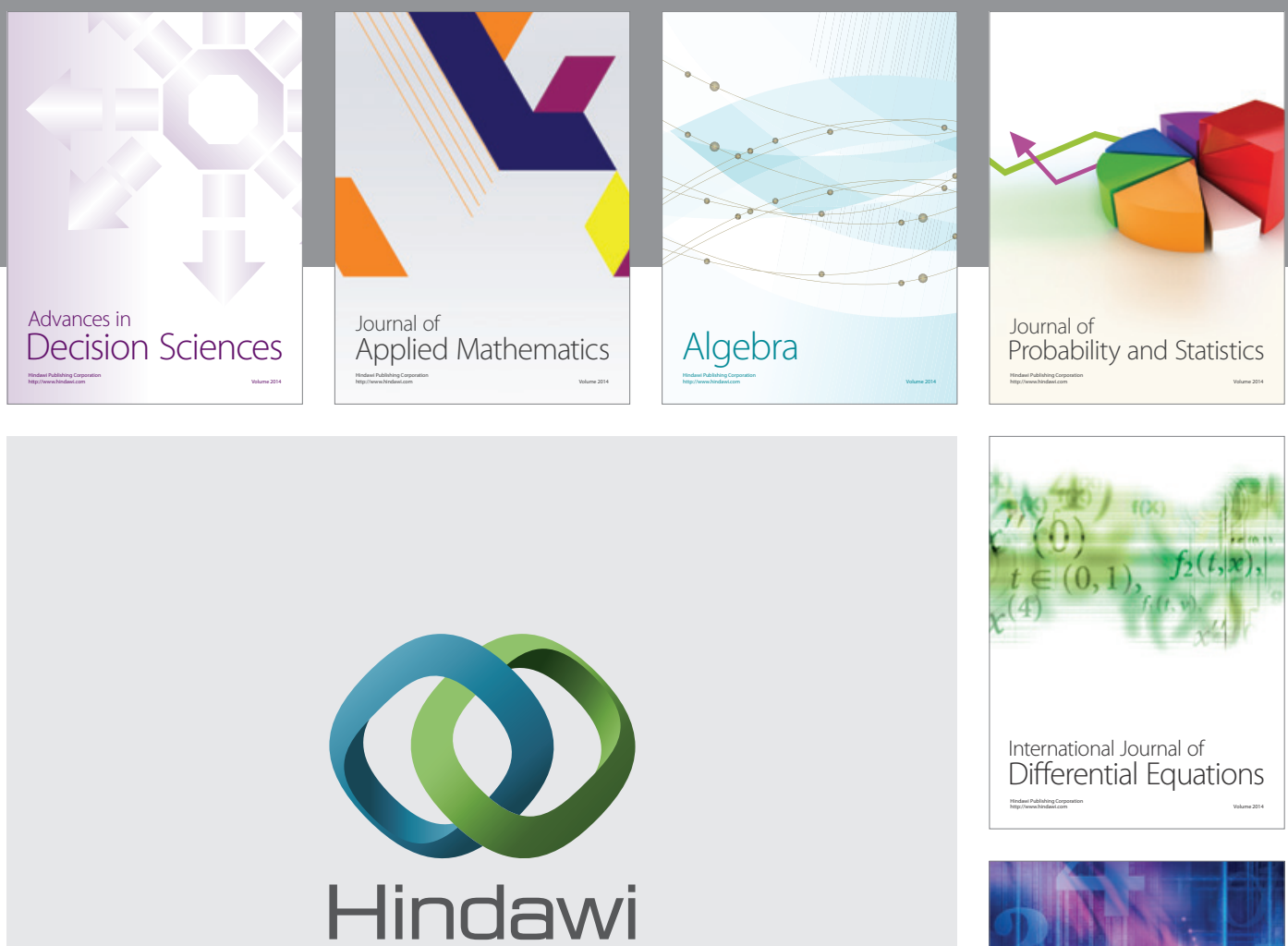

Submit your manuscripts at http://www.hindawi.com
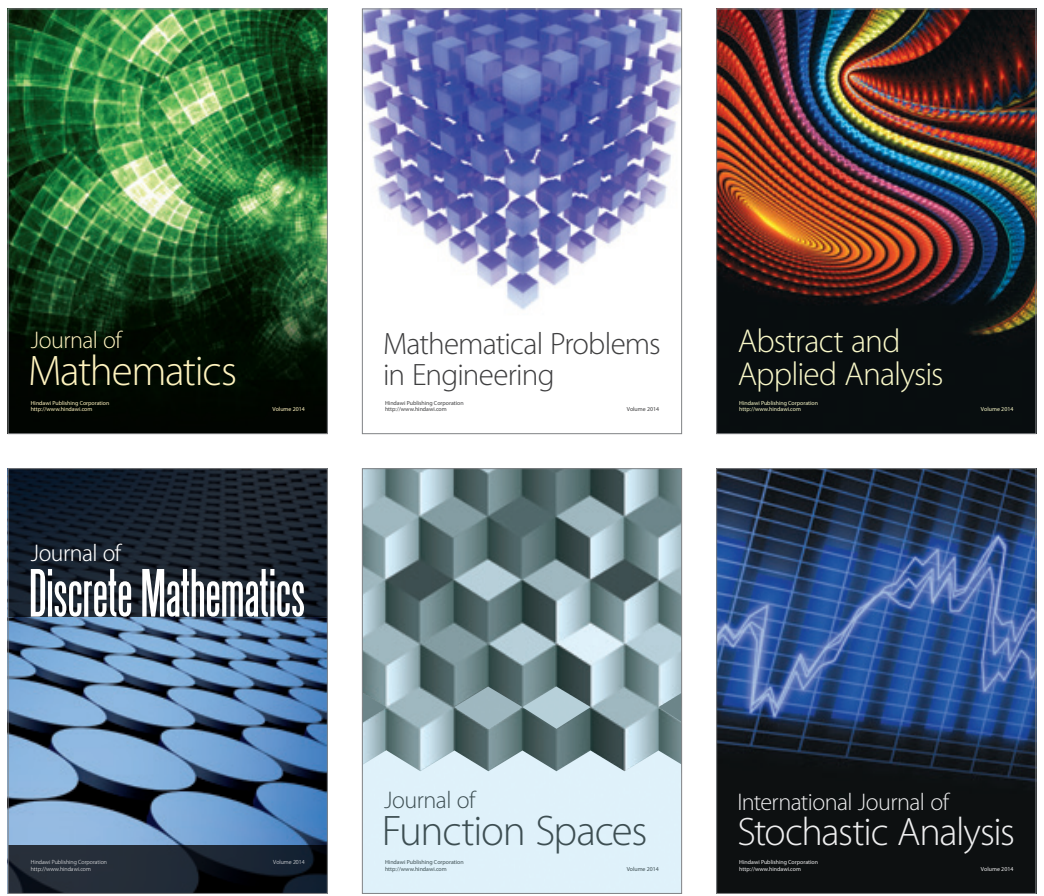

Journal of

Function Spaces

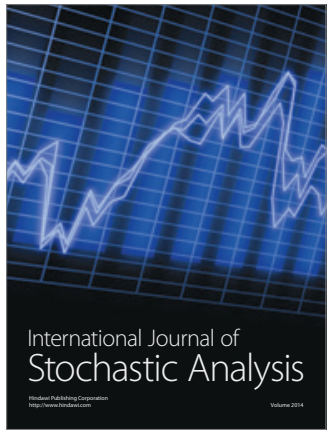

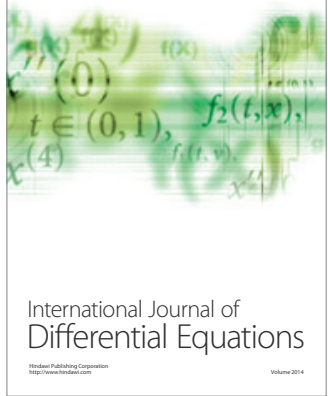
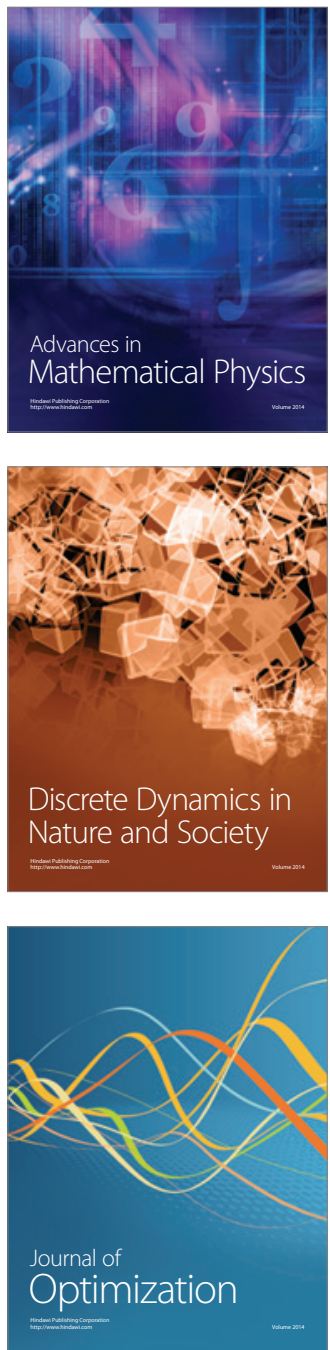